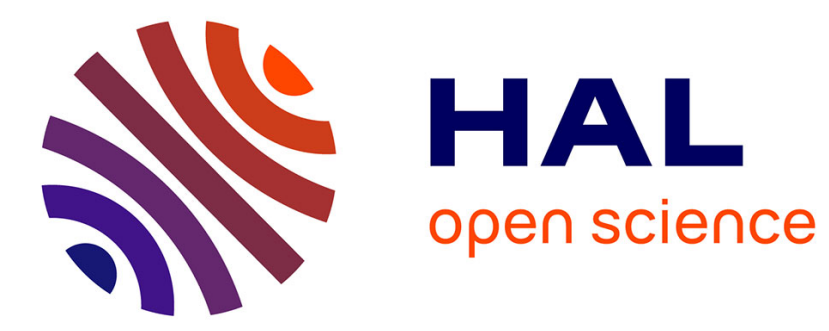

\title{
Consistent estimation of species abundance from a presence-absence map
}

Christine H. Müller, Richard Huggins, Wen-Han Hwang

\section{To cite this version:}

Christine H. Müller, Richard Huggins, Wen-Han Hwang. Consistent estimation of species abundance from a presence-absence map. Statistics and Probability Letters, 2011, 81 (9), pp.1449. 10.1016/j.spl.2011.04.005 . hal-00762904

\section{HAL Id: hal-00762904 https://hal.science/hal-00762904}

Submitted on 9 Dec 2012

HAL is a multi-disciplinary open access archive for the deposit and dissemination of scientific research documents, whether they are published or not. The documents may come from teaching and research institutions in France or abroad, or from public or private research centers.
L'archive ouverte pluridisciplinaire HAL, est destinée au dépôt et à la diffusion de documents scientifiques de niveau recherche, publiés ou non, émanant des établissements d'enseignement et de recherche français ou étrangers, des laboratoires publics ou privés. 


\section{Accepted Manuscript}

Consistent estimation of species abundance from a presence-absence

map

Christine H. Müller, Richard Huggins, Wen-Han Hwang

PII:

S0167-7152(11)00136-2

DOI:

10.1016/j.spl.2011.04.005

Reference: $\quad$ STAPRO 5980

To appear in: Statistics and Probability Letters

Received date: 3 November 2010

Revised date: 31 March 2011

Accepted date: 12 April 2011

Please cite this article as: Müller, C.H., Huggins, R., Hwang, W.-H., Consistent estimation of species abundance from a presence-absence map. Statistics and Probability Letters (2011), doi:10.1016/j.spl.2011.04.005

This is a PDF file of an unedited manuscript that has been accepted for publication. As a service to our customers we are providing this early version of the manuscript. The manuscript will undergo copyediting, typesetting, and review of the resulting proof before it is published in its final form. Please note that during the production process errors may be discovered which could affect the content, and all legal disclaimers that apply to the journal pertain. 


\title{
Consistent estimation of species abundance from a presence-absence map
}

\author{
Christine H. Müller \\ TU Dortmund University, Germany \\ Richard Huggins, \\ The University of Melbourne, Australia \\ Wen-Han Hwang \\ National Chung Hsing University, Taiwan
}

March 31, 2011

\begin{abstract}
The estimation of the abundance of a species using the presence or absence of the species over a grid of cells simplifies data collection but the resulting statistical analysis is challenging. Several estimators have been proposed but their properties are unknown. Here we consider a generalized gamma-Poisson model which allows dependencies across the grid and develop a new estimator for this model. It is shown that this estimator is consistent, allowing us to conclude that it is indeed possible to estimate abundance from presence-absence maps.
\end{abstract}

Keywords: Presence Absence Map; Consistency; Gamma-Poisson Model.

\section{Introduction}

In ecological studies where the interest is in estimating the number of individuals of a certain species in a given area it is often too laborious to count all individuals in an area. A more labor effective procedure is to divide the area in $M$ cells and to determine for each cell whether the species is present or not. The data may be thought of as a presence-absence map that identifies the occupied cells and it is often represented by a $I \times J$-matrix $\left(y_{i j}\right)_{i=1, \ldots, I, j=1, \ldots, J}$ with $M=I \cdot J$ cells, where $y_{i j}=1$ if the $i, j$ cell is unoccupied cell and 0 otherwise. Then the question is whether it is possible to estimate the total number $N$ of the species in the area from these data in general. The analysis of these data presents an interesting statistical challenge (Kunin 1998, He and Gaston 2000, Kunin et al. 2000, Conlinsk et al. 2007, He and Gaston 2007). 
The resolution of the problem requires distributional assumptions on the unknown numbers $X_{i j}$ of individuals in each cell. Suppose that there are $N$ individuals so that $N=\sum_{i=1}^{I} \sum_{j=1}^{J} X_{i j}$. The simplest approach is to assume that each individual is independent of other individuals and the probability of its occurrence in a given cell is $1 / M$. Then $\left(X_{i j}\right)_{i=1, \ldots, I, j=1, \ldots, J}$ has a multinomial distribution where each cell has the same probability and the abundance $N$ can be estimated using maximum likelihood. Alternatively, one may suppose that $X_{i j}$ are independently and identically distributed with a common Poisson distribution with mean $\lambda$. In this case, the probability a cell is empty is $e^{-\lambda}$. Let $m_{0}=\sum_{i=1}^{I} \sum_{j=1}^{J} y_{i j}$ be the number of empty (unoccupied) cells. Then by the method of moments, we may estimate $\lambda$ by $\log \left(M / m_{0}\right)$ so that an estimate of $N$ is $M \log \left(M / m_{0}\right)$. However, since the assumption of independently and identically distributed random variables does not reflect the spatial aggregation (clustering) typically observed in nature, this method often underestimates the true abundance (He and Gaston 2000) and hence is not recommended in literature.

In order to reflect the spatial aggregation, He and Gaston (2000) used the negative binomial distribution, i.e., they assumed that the $X_{i j}$ follow the negative binomial distribution. Although it is well known that the negative binomial distribution is more appropriate than the Poisson distribution for modeling aggregated organisms and populations (Pielou 1977), estimation is more difficult as there are two parameters in the model and they are not identifiable from a presence-absence map. To circumvent this difficulty, He and Gaston (2000) generate a coarser map, where adjacent pairs of cells are merged from the base map and impose a constraint on the clumping parameter of the coarser map. The resulting estimator for the species abundance was shown to outperform other methods in several empirical studies (He and Gaston 2000, 2007). Nevertheless, He and Gaston (2000, 2007) do not provide theoretical justification for the constraint and this weakness is criticized by Conlisk et al. (2007). By noting that the negative binomial distribution arises from a gamma-Poisson mixture model, Hwang and He (2011) proposed a generalized gamma-Poisson mixture model. In this model, a gamma random variable is first associated with each cell, as in the negative binomial model, but correlations between the cells are modelled by allowing the conditional mean of the Poisson counts to be a weighted sum of all the gamma random variables. Hwang and He (2011) used some gamma approximations to find an estimator. When applied to forest census data (Condit et al., 1996), they showed that the estimator based on the generalized gamma Poisson model could improve estimation of abundance from occurrence data. To date, however, no statistical properties of the estimators of He and Gaston (2000) and Hwang and He (2011) are known. Even consistency has not been proven.

In this study, we develop a new consistent estimator under the generalised gammaPoisson model. In Section 2 we reformulate the model of He and Gaston (2000) using the gamma-Poisson framework and then describe the generalised gamma-Poisson model. In Section 3 we derive the proposed estimator for the generalised gammaPoisson model whose consistency is shown in Section 4. This consistency proof is not straightforward and needs some tricky arguments which are given in the Appendix. The estimator is applied to real data in Section 5. The results are discussed in Section 6 . 


\section{Model}

For simplicity, assume that $I$ and $J$ are even. Let $\lambda_{i j}$ be the mean abundance at the cell $(i, j)$. This mean may depend on environmental characteristics, such as the slope of the land, the soil content and the humidity gradient. Given the means $\lambda_{i j}$, we assume that the abundances $X_{i j}$ follow Poisson distributions and they are independent of each other. If the $\lambda_{i j}$ are independent and identically distributed gamma distributed random variables with parameters $k$ and $\beta$ so that $\mathrm{E}\left(\lambda_{i j}\right)=k \beta$ and $\operatorname{var}\left(\lambda_{i j}\right)=k \beta^{2}$, then $X_{i j}$ are independent and each $X_{i j}$ has a negative binomial distribution. The parameter $k$ is called the clumping parameter (Pielou, 1977) as it corresponds to the degree of aggregation of individuals in a cell. It is easy to see the absence probability $P\left(X_{i j}=0\right)$ is $1 /(1+\beta)^{k}$. As a consequence, the likelihood function based on the observed $y_{i j}$ is

$$
\left\{\frac{1}{(1+\beta)^{k}}\right\}^{m_{0}}\left\{1-\frac{1}{(1+\beta)^{k}}\right\}^{M-m_{0}} .
$$

The likelihood is not identifiable as there are two unknown parameters but only a single statistic $m_{0}$. In order to estimate the parameters, He and Gaston (2000) create a coarser map where adjacent cells in the original base map are merged, collapsing a $2 \times 2$ cell to a single cell. That is, let $X_{(2 i-1)(2 j-1)}^{q}=X_{(2 i-1)(2 j-1)}+$ $X_{(2 i-1)(2 j)}+X_{(2 i)(2 j-1)}+X_{(2 i)(2 j)}$, for $i=1, \cdots, I / 2$ and $j=1, \cdots, J / 2$, which is the number of individuals in the merged quadruple cells. The coarser quad map then indicates whether the $X_{(2 i-1)(2 j-1)}^{q}$ is larger than zero or not. Denote by $m_{0}^{q}$ the number of empty cells in the coarser map. Noting $N=M k \beta$, He and Gaston (2000) solved the equations $m_{0} / M=P\left(X_{i j}=0\right)$ and $4 m_{0}^{q} / M=P\left(X_{i j}^{q}=0\right)$ to estimate $N$ and $k$. Simple algebra shows that $X_{(2 i-1)(2 j-1)}^{q}$ has a negative binomial distribution with the clumping parameter $4 k$. Nevertheless, He and Gaston (2000) suppose that $X_{(2 i-1)(2 j-1)}^{q}$ has the same distribution as $X_{i j}$, which has clumping parameter $k$ and mean $k \beta$. This presumption is mainly based on empirical evidence and obviously contradicts the theoretical calculations. In addition, Conlisk (2007) showed that there is no solution from the negative binomial model under the correct distribution. To overcome the weaknesses of this approach, Hwang and He (2011) proposed an extension in the gamma-Poisson model framework. This extension yields a generalized gamma-Poisson mixtured model that has meaning in the ecological context.

To develop this model, recall that the mean level $\lambda_{i j}$ represents the environmental characteristics of the immediate area of study, hence the variables $\lambda_{i j}$ would tend to vary smoothly over nearby cells. Hence, it is unrealistic to assume independence of the $\lambda_{i j}$. It is more appropriate to consider the spatial correlation of the $\lambda_{i j}$ over the cells so that the $\lambda_{i j}$ in neighboring cells are more similar than between those that are further apart. This observation motivated Hwang and He (2011) to model the mean levels as a gamma random field. Formally, let $V_{s t}, s, t \in \mathbb{Z}=\{0,-1,1,-2,2, \ldots\}$, be independent and identically gamma distributed random variables with parameters $a$ and $b$ (so that $\mathrm{E}\left(V_{s t}\right)=a b$ and $\left.\operatorname{var}\left(V_{s t}\right)=a b^{2}\right)$. Given $0 \leq \rho<1$, we define the mean levels

$$
\lambda_{i j}=\sum_{s=-\infty}^{\infty} \sum_{t=-\infty}^{\infty} \rho^{|i-s|+|j-t|} V_{s t}
$$


which is a spatial moving average of the $V_{s t}$. The parameter $\rho$ reflects the strength of the spatial correlation of the mean levels $\lambda_{i j}$. It is then assumed that the conditional distributions of the $X_{i j}$ given $V_{s t}=v_{s t}$ for all $s, t \in \mathbb{Z}$ are Poisson with mean $\lambda_{i j}$ and that the $X_{i j}$ and $X_{k l}$ are conditionally independent given $V_{s t}=v_{s t}, s, t \in \mathbb{Z}$ for $(i, j) \neq(k, l)$. As the joint distribution of $X_{i j}$ reduces to the usual independent negative binomial model when $\rho=0$, it is called the generalized gamma-Poisson mixture model. Hwang and He (2011) used gamma approximations to find an estimator, however, the consistency of this estimator remains an open question. Here we propose a new moment estimator whose consistency can be established.

\section{Estimation}

Since the conditional distribution of $X_{i j}$ given $V_{s t}=v_{s t}, s, t \in \mathbb{Z}$, is Poisson with parameter $\lambda_{i j}=\sum_{s=-\infty}^{\infty} \sum_{t=-\infty}^{\infty} \rho^{|i-s|+|j-t|} v_{s t}$, we see that

$$
\mathrm{E}\left(X_{i j}\right)=\sum_{s=-\infty}^{\infty} \sum_{t=-\infty}^{\infty} \rho^{|i-s|+|j-t|} \mathrm{E}\left(V_{s t}\right)=\sum_{s=-\infty}^{\infty} \rho^{|i-s|} \sum_{t=-\infty}^{\infty} \rho^{|j-t|} a b=\left(\frac{1+\rho}{1-\rho}\right)^{2} a b,
$$

and thus

$$
\mathrm{E}(N)=\sum_{i=1}^{I} \sum_{j=1}^{J} E\left(X_{i j}\right)=I J\left(\frac{1+\rho}{1-\rho}\right)^{2} a b .
$$

Once we have estimators $\widehat{a}, \widehat{b}, \widehat{\rho}$ of $a, b$, and $\rho$ this yields the estimator

$$
\widehat{N}=I J\left(\frac{1+\widehat{\rho}}{1-\widehat{\rho}}\right)^{2} \widehat{a} \widehat{b}
$$

and our main task is to estimate these parameters using presence-absence data.

Let $Y_{i j}=1_{\{0\}}\left(X_{i j}\right)$ be an indicator of whether the cell $(i, j)$ is empty or not. The variables $Y_{i(2 j-1)}^{d}=Y_{i(2 j-1)} Y_{i(2 j)}$ and $Y_{(2 i-1)(2 j-1)}^{q}=Y_{(2 i-1)(2 j-1)} Y_{(2 i-1)(2 j)} Y_{(2 i)(2 j-1)} Y_{(2 i)(2 j)}$ indicate whether the double and quadruple cells are empty. Our approach is based on the method of moments and to this end we need:

\section{Proposition 1}

$$
\begin{aligned}
& \text { a) } E\left(Y_{i j}\right)=\prod_{s=-\infty}^{\infty} \prod_{t=-\infty}^{\infty}\left(1+b \rho^{|i-s|+|j-t|}\right)^{-a}, \\
& \text { b) } \quad E\left(Y_{i j}^{d}\right)=\prod_{s=-\infty}^{\infty} \prod_{t=-\infty}^{\infty}\left(1+b \rho^{|i-s|}\left(\rho^{|j-t|}+\rho^{|j+1-t|}\right)\right)^{-a}, \\
& \text { c) } E\left(Y_{i j}^{q}\right) \\
& =\prod_{s=-\infty}^{\infty} \prod_{t=-\infty}^{\infty}\left(1+b\left(\rho^{|i-s|}+\rho^{|i+1-s|}\right)\left(\rho^{|j-t|}+\rho^{|j+1-t|}\right)\right)^{-a} .
\end{aligned}
$$


Define $M_{0}=\sum_{i=1}^{I} \sum_{j=1}^{J} Y_{i j}, \quad M_{0}^{d}=\sum_{i=1}^{I} \sum_{j=1}^{J / 2} Y_{i(2 j-1)}^{d}$ and $M_{0}^{q}$ $=\sum_{i=1}^{I / 2} \sum_{j=1}^{J / 2} Y_{(2 i-1)(2 j-1)}^{q}$ with realizations $m_{0}, m_{0}^{d}$, and $m_{0}^{q}$. Then, $a, b$, and $\rho$ can be estimated by solving

$$
\begin{aligned}
& \frac{1}{I J} m_{0}=\mathrm{E}_{(a, b, \rho)}\left(\frac{1}{I J} M_{0}\right)=\prod_{s=-\infty}^{\infty} \prod_{t=-\infty}^{\infty}\left(1+b \rho^{|s|+|t|}\right)^{-a}, \\
& \frac{2}{I J} m_{0}^{d}=\mathrm{E}_{(a, b, \rho)}\left(\frac{2}{I J} M_{0}^{d}\right)=\prod_{s=-\infty}^{\infty} \prod_{t=-\infty}^{\infty}\left(1+b \rho^{|s|}\left(\rho^{|t|}+\rho^{|t+1|}\right)\right)^{-a}, \\
& \frac{4}{I J} m_{0}^{q}=\mathrm{E}_{(a, b, \rho)}\left(\frac{4}{I J} M_{0}^{q}\right)=\prod_{s=-\infty}^{\infty} \prod_{t=-\infty}^{\infty}\left(1+b\left(\rho^{|s|}+\rho^{|s+1|}\right)\left(\rho^{|t|}+\rho^{|t+1|}\right)\right)^{-a} .
\end{aligned}
$$

\section{Consistency}

To establish consistency we let $I J \rightarrow \infty$ so that the number of cells observed increases. As we suppose the occupancy probabilities do not depend on $I$ or $J$ this implies that $N \rightarrow \infty$. This corresponds to increasing the size of the map.

Theorem 1 If $(a, b, \rho) \in(0, \infty) \times(0, \infty) \times[0,1)$, then $\left(\frac{1}{I J} M_{0}, \frac{2}{I J} M_{0}^{d}, \frac{4}{I J} M_{0}^{q}\right)^{\top}$ is a weakly consistent estimator of $\left(E_{(a, b, \rho)}\left(\frac{1}{I J} M_{0}\right), E_{(a, b, \rho)}\left(\frac{2}{I J} M_{0}^{d}\right), E_{(a, b, \rho)}\left(\frac{4}{I J} M_{0}^{q}\right)\right)^{\top}$ as $I J \rightarrow \infty$.

The consistency of the estimators $(\widehat{a}, \widehat{b}, \widehat{\rho})$ and thus of $\widehat{N}$ follows from the following general proposition, which is similar to those of Amemiya (1973, Lemma 3), White (1980, Lemma 2.2) and White (1981, Theorem 2.1).

Proposition 2 Let be $\Theta \subset \mathbb{R}^{L}$ compact, $g: \Theta \rightarrow \mathbb{R}^{L}$ continuous satisfying $g(\theta) \neq$ $g\left(\theta^{\prime}\right)$ for all $\theta, \theta^{\prime} \in \Theta$ with $\theta \neq \theta^{\prime}$, and $X_{N}$ an $L$ dimensional random vector. If $X_{N}$ is a weakly consistent estimator for $g(\theta)$, then

$$
\widehat{\theta}_{N} \in \arg \min _{\theta \in \Theta}\left\|X_{N}-g(\theta)\right\|
$$

is a weakly consistent estimator of $\theta$.

Since $\mathrm{E}(N)$ defined in $(1)$ is a continuous function of $(a, b, \rho)$, Theorem 1 implies at once:

Theorem 2 The abundance estimator $\widehat{N}$ given by (2), where $(a, b, \rho) \in \Theta$ for $a$ compact parameter space $\Theta \subset(0, \infty) \times(0, \infty) \times(0,1)$ are solutions of (3), satisfies $\widehat{N}-E(N)=o_{P}(I J)$.

Hence we need only to prove Theorem 1. This proof is not straightforward because of the dependence structure and therefore given together with the proof of Proposition 1 in the Appendix. 


\section{Application to real data}

We consider data from a complete census of a tropical rain forest in Barro Colorado Island (BCI), Panama (Condit et al. 1996). The study area was a 50-hectare $(500 \times 1000 \mathrm{~m})$ rectangle plot referred to as the BCI plot. The tree plot was established in 1980 and has been surveyed six times so far. The data from the 1985 census are used in this study. The data records the exact location of species for each free-standing tree with diameter at breast height (dbh) at least $1 \mathrm{~cm}$ in the plot. In the 1985 census, there are 238,018 trees representing 299 species. We illustrate the computation of the estimator on the species Faramea occidentalis. Here $N=25094$, $m_{0}=7824, m_{0}^{d}=1994$ and $m_{0}^{q}=357$. There were $20000-7824=12176$ occupied cells, giving a lower bound on $N$. We solved the estimating equations (3) with the products from negative to positive infinity replaced by products from $-T$ to $T$ by minimizing an objective function consisting of the sums of squares of the differences. We considered $\rho=0.05,0.06, \ldots, 0.99$ and for each value of $\rho$ we found values of $a$ and $b$ to minimize the objective function. In Figure 1 we plot the value of the minimum and the estimated $\widehat{N}$ as functions of $\rho$ for $T=60$. The minimum was quite flat as a function of $\rho$ near the minimum, however, a plot of the logarithm of the minimum, not given here, did show a distinct minimum. At this minimum, $\hat{a}=0.00066, \hat{b}=3.27$, and $\hat{\rho}=0.92$ and the estimated abundance was $\widehat{N}=24774$. In the second part of the figure we see that the estimated value of $N$ is also quite flat and is close to the true value for a range of values of $\rho$.
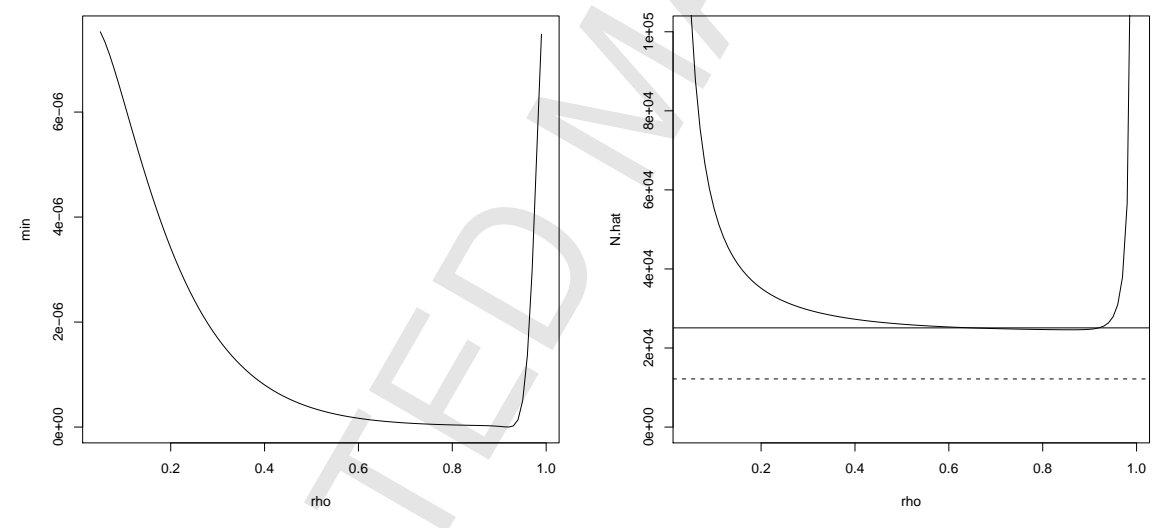

Figure 1: The minimum value of the sums of squared differences of (3) and $\widehat{N}$ as functions of $\rho \in(0,1)$ for Faramea occidentalis. In the second plot, the horizontal line gives the known value of $N$ (solid line) and the lower bound on $N$ (dashed line).

\section{Discussion}

We have demonstrated analytically that the new estimator is consistent and conclude that for the mixed gamma-Poisson model it is possible to estimate abundance from presence-absence maps in a quite general setting. 
The assumption of a compact parameter space is required to prove Proposition 2 and hence Theorem 2, but since the compact parameter space can be arbitrarily large, this is not a strong restriction. We also require $g(\theta) \neq g\left(\theta^{\prime}\right)$ for all $\theta, \theta^{\prime} \in \Theta$ with $\theta \neq \theta^{\prime}$. This is an identifiability condition which is usually difficult to verify in nonlinear settings with more than two parameters. Here this means that the solutions $\widehat{a}, \widehat{b}, \widehat{\rho}$ of the equations given by (3) are unique, i.e. $(a, b, \rho)$ is identified by $\left(\frac{1}{I J} m_{0}, \frac{2}{I J} m_{0}^{d}, \frac{4}{I J} m_{0}^{q}\right)^{\top}$. This is not the case for $\rho=0$ or $a=0$ or $b=0$, but we conjecture that identifiability holds for $\rho>0, a>0, b>0$. Since $\rho=0$ does not satisfy the conditions of the Theorem, we have shown consistency only in the case where the cell counts are dependent. Moreover, consistency is obtained as $I J \rightarrow \infty$ with the cell occupancy probabilities remaining the same so that $N \rightarrow \infty$. An alternate approach not considered here would be to allow the grid to become finer so that $N$ is the number of individuals in a given area. In this case, the large sample properties are problematic as for a fine enough grid, $N$ will be the total number of occupied cells.

We were not concerned with the practical application of the new estimator but have focused on consistency. In particular we have not addressed the goodness of fit of the model. The precision of the estimator may be addressed using bootstrap methods according to Hall 1988.

\section{References}

[1] Amemiya, T. (1973). Regression analysis when the dependent variable is truncated normal. Econometrica 41, 997-1016.

[2] Condit, R., Hubbell, S. P. and Foster, R. B. (1996). Changes in a tropical forest with a shifting climate: results from a 50 ha permanent census plot in Panama. Journal of Tropical Ecology 12, 231-256.

[3] Conlisk, E., Conlisk, J., and Harte, J. (2007). The impossibility of estimating a negative binomial clustering parameter from presence-absence data: A comment on He and Gaston. The American Naturalist 170, 651-654.

[4] Hall, P. (1988) On confidence intervals for spatial parameters estimated from nonreplicated data. Biometrics, 44, 271-277.

[5] He, F. and Gaston, K.J. (2000). Estimating species abundance from occurrence. The American Naturalist 156, 553-559.

[6] He, F. and Gaston, K.J. (2007). Estimating abundance from occurrence: An underdetermined problem. The Ammerican Naturalist, 170, 655-659.

[7] Hwang, W.H. and He, F. (2011). Estimating abundance from presence-absence maps. Methods in Ecology and Evolution (in press).

[8] Kunin, W.E. (1998). Extrapolating species abundance across spatial scales. Science 281, 1513-1515. 
[9] Kunin, W.E., Hartley, S., and Lennon, J.J. (2000). Scaling down: On the challenge of estimating abundance from occurrence patterns. The American Naturalist 156, 560-566.

[10] Pielou, E.C. (1977). Mathematical Ecology. John Wiley, New York.

[11] White, H. (1980). Nonlinear regression on cross-section data. Econometrica 48, 721-746.

[12] White, H. (1981). Consequences and detection of misspecified nonlinear regression models. J. Amer. Statist. Assoc. 76, 419-433.

\section{A Proofs}

To prove Theorem 1, some propositions must be proved. As Proposition 1 and Proposition 3 below are related we combine their proofs.

\section{Proposition 3}

a) $\quad E\left(Y_{i j} \cdot Y_{k l}\right)=\prod_{s=-\infty}^{\infty} \prod_{t=-\infty}^{\infty}\left(1+b\left[\rho^{|i-s|+|j-t|}+\rho^{|k-s|+|l-t|}\right]\right)^{-a}$

b) $\quad E\left(Y_{i j}^{d} \cdot Y_{k l}^{d}\right)$

$$
=\prod_{s=-\infty}^{\infty} \prod_{t=-\infty}^{\infty}\left(1+b\left[\rho^{|i-s|}\left(\rho^{|j-t|}+\rho^{|j+1-t|}\right)+\rho^{|k-s|}\left(\rho^{|l-t|}+\rho^{|l+1-t|}\right)\right]\right)^{-a},
$$

c) $\quad E\left(Y_{i j}^{q} \cdot Y_{k l}^{q}\right)$

$$
\begin{aligned}
=\prod_{s=-\infty}^{\infty} \prod_{t=-\infty}^{\infty}(1 & +b\left[\left(\rho^{|i-s|}+\rho^{|i+1-s|}\right)\left(\rho^{|j-t|}+\rho^{|j+1-t|}\right)\right. \\
& \left.\left.+\left(\rho^{|k-s|}+\rho^{|k+1-s|}\right)\left(\rho^{|l-t|}+\rho^{|l+1-t|}\right)\right]\right)^{-a} .
\end{aligned}
$$

\section{Proof of Propositions 1 and 3.}

Since a Poisson random variable $X$ with parameter $\lambda$ satisfies $P(X=0)=\exp \{-\lambda\}$, using the moment generating function of the gamma distribution and the independence of the $V_{s t}$, we have

$$
\begin{aligned}
& E\left(Y_{i j}\right)=P\left(X_{i j}=0\right) \\
& =\prod_{s=-\infty}^{\infty} \prod_{t=-\infty}^{\infty} \mathrm{E}\left(\exp \left\{-\rho^{|i-s|+|j-t|} V_{s t}\right\}\right)=\prod_{s=-\infty}^{\infty} \prod_{t=-\infty}^{\infty}\left(1+b \rho^{|i-s|+|j-t|}\right)^{-a}
\end{aligned}
$$

which is Proposition 1 a). Since $X_{i j}$ and $X_{k l}$ are conditionally independent given $V_{s t}$ with $s, t \in \mathbb{Z}$, Proposition $1 \mathrm{~b}$ ) and Proposition 3 a) follow from

$$
\begin{aligned}
& E\left(Y_{i j} \cdot Y_{k l}\right)=P\left(X_{i j}=0, X_{k l}=0\right) \\
& =\mathrm{E}\left(\exp \left\{-\sum_{s=-\infty}^{\infty} \sum_{t=-\infty}^{\infty}\left(\rho^{|i-s|+|j-t|}+\rho^{|k-s|+|l-t|}\right) V_{s t}\right\}\right)
\end{aligned}
$$


Proposition 1 c) is a special case of Proposition 3 b). Since b) and c) of Proposition 3 can be proved similarly, only the proof of c) is shown here:

$$
\begin{aligned}
& E\left(Y_{i j}^{q} \cdot Y_{k l}^{q}\right) \\
& =P\left(X_{i j}=0, X_{i(j+1)}=0, X_{(i+1) j}=0, X_{(i+1)(j+1)}=0\right. \\
& \left.\quad X_{k l}=0, X_{k(l+1)}=0, X_{(k+1) l}=0, X_{(k+1)(l+1)}=0\right) \\
& \quad E\left(\operatorname { e x p } \left[-\sum_{s=-\infty}^{\infty} \sum_{t=-\infty}^{\infty}\left(\rho^{|i-s|+|j-t|}+\rho^{|i-s|+|j+1-t|}+\rho^{|i+1-s|+|j-t|}+\rho^{|i+1-s|+|j+1-t|}\right.\right.\right. \\
& \left.\left.\left.\quad+\rho^{|k-s|+|l-t|}+\rho^{|k-s|+|l+1-t|}+\rho^{|k+1-s|+|l-t|}+\rho^{|k+1-s|+|l+1-t|}\right) V_{s t}\right]\right) \cdot \square
\end{aligned}
$$

Proposition 4 Let be $(a, b, \rho) \in(0, \infty) \times(0, \infty) \times[0,1)$. Then there exists a constant $c$ only depending on $a, b, \rho$ such that

$$
\begin{aligned}
& \sum_{i=1}^{I} \sum_{i=1}^{I} \sum_{j=1}^{J} \sum_{j=1}^{J} \ln \left(\frac{E\left(Y_{i j} Y_{k l}\right)}{E\left(Y_{i j}\right) E\left(Y_{k l}\right)}\right) \leq c I J, \\
& \sum_{i=1}^{I} \sum_{i=1}^{I} \sum_{j=1}^{J / 2} \sum_{j=1}^{J / 2} \ln \left(\frac{E\left(Y_{i(2 j-1)}^{d} Y_{k(2 l-1)}^{d}\right)}{E\left(Y_{i(2 j-1)}^{d}\right) E\left(Y_{k(2 l-1)}^{d}\right)}\right) \leq c I J, \\
& \sum_{i=1}^{I / 2} \sum_{i=1}^{I / 2} \sum_{j=1}^{J / 2} \sum_{j=1}^{J / 2} \ln \left(\frac{E\left(Y_{(2 i-1)(2 j-1)}^{q} Y_{(2 k-1)(2 l-1)}^{q}\right)}{E\left(Y_{(2 i-1)(2 j-1)}^{q}\right) E\left(Y_{(2 k-1)(2 l-1)}^{q}\right)}\right) \leq c I J .
\end{aligned}
$$

To prove Proposition 4, we need

Lemma 1 Let $\alpha_{i}(s)=\rho^{|i-s|}+\rho^{|i+1-s|}$ for $i=1, \ldots, I$. Then

$$
\sum_{i=1}^{I} \sum_{k=1}^{I} \sum_{s=-\infty}^{\infty} \alpha_{i}(s) \alpha_{k}(s) \leq \begin{cases}12 I, & \text { for } \rho=0, \\ \frac{1}{\left(1-\rho^{2}\right)(1-\rho)^{4}}\left(4 \cdot 42 I+\frac{4}{\rho} 20 I\right), & \text { for } \rho \in(0,1),\end{cases}
$$

for all $I \in \mathbb{N}$.

\section{Proof.}

If $\rho \in(0,1)$, then $\alpha_{i}(s)=\rho^{i-s}+\rho^{i+1-s}=\rho^{i-s}(1+\rho) \leq 2 \rho^{i-s}$ for $s \leq i$ and $\alpha_{i}(s)=\rho^{s-i}+\rho^{s-(i+1)}=\rho^{s-i-1}(\rho+1) \leq 2 \rho^{s-i-1}$ for $s \geq i+1$. Analogous bounds hold for $\alpha_{k}(s)$. Let $i \leq k$. Then

$$
\begin{aligned}
& \sum_{s=-\infty}^{\infty} \alpha_{i}(s) \alpha_{k}(s) \\
& \leq \sum_{s=-\infty}^{i}\left(2 \rho^{i-s}\right)\left(2 \rho^{k-s}\right)+\sum_{s=i+1}^{k}\left(2 \rho^{s-i-1}\right)\left(2 \rho^{k-s}\right)+\sum_{s=k+1}^{\infty}\left(2 \rho^{s-i-1}\right)\left(2 \rho^{s-k-1}\right) \\
& =4\left[\rho^{k-i} \sum_{s=0}^{\infty}\left(\rho^{2}\right)^{s}+\rho^{k-i-1}(k-i)+\rho^{k-i} \sum_{s=0}^{\infty}\left(\rho^{2}\right)^{s}\right] \\
& =4\left[\rho^{k-i} \frac{1}{1-\rho^{2}}+\rho^{k-i-1}(k-i)+\rho^{k-i} \frac{1}{1-\rho^{2}}\right] \\
& \leq 4 \frac{\rho^{|k-i|-1}}{1-\rho^{2}}(2 \rho+|k-i|) .
\end{aligned}
$$


Similarly, the same upper bound holds for $i \geq k$. As

$$
\begin{gathered}
\sum_{i=1}^{I} \sum_{k=1}^{I} \rho^{|k-i|}|k-i|=\frac{2}{(1-\rho)^{4}}\left((I-1) \rho-2 I \rho^{2}+(I+1) \rho^{3}+(I+1) \rho^{I+1}\right. \\
\left.-2 I \rho^{I+2}+(I-1) \rho^{I+3}\right) \leq \frac{2}{(1-\rho)^{4}} 10 I
\end{gathered}
$$

we see that

$$
\begin{aligned}
& \sum_{i=1}^{I} \sum_{k=1}^{I} \sum_{s=-\infty}^{\infty} \alpha_{i}(s) \alpha_{k}(s) \\
& \quad \leq \sum_{i=1}^{I} \sum_{k=1}^{I} 4 \frac{\rho^{|k-i|-1}}{1-\rho^{2}}(2 \rho+|k-i|) \\
& \quad \leq \frac{4}{1-\rho^{2}}\left(2 \sum_{i=1}^{I} \sum_{k=1}^{I} \rho^{|k-i|}|k-i|+2 I+\frac{1}{\rho} \sum_{i=1}^{I} \sum_{k=1}^{I} \rho^{|k-i|}|k-i|\right) \\
& \quad \leq \frac{4}{1-\rho^{2}}\left(2 \frac{2}{(1-\rho)^{4}} 10 I+2 I+\frac{1}{\rho} \frac{2}{(1-\rho)^{4}} 10 I\right) \\
& \quad \leq \frac{1}{\left(1-\rho^{2}\right)(1-\rho)^{4}}\left(4 \cdot 42 I+\frac{4}{\rho} 20 I\right)
\end{aligned}
$$

If $\rho=0$, we obtain using $0^{0}=1$

$$
\sum_{s=-\infty}^{\infty} \alpha_{i}(s) \alpha_{k}(s) \leq \begin{cases}4, & \text { for }|k-i| \leq 1 \\ 0, & \text { for }|k-i|>1\end{cases}
$$

as required.

\section{Proof of Proposition 4.}

To complete the proof of Proposition 4, let

$$
\begin{aligned}
\alpha_{i}^{0}(s) & =\rho^{|i-s|}, & \alpha_{i}(s) & =\rho^{|i-s|}+\rho^{|i+1-s|}, \\
\beta_{j}^{0}(t) & =\rho^{|j-t|}, & \beta_{j}(t) & =\rho^{|j-t|}+\rho^{|j+1-t|} .
\end{aligned}
$$

Setting

$$
\begin{aligned}
\alpha_{i}^{1}(s)=\alpha_{i}^{0}(s), & \beta_{j}^{1}(t)=\beta_{j}^{0}(t), \\
\alpha_{i}^{2}(s)=\alpha_{i}^{0}(s), & \beta_{j}^{2}(t)=\beta_{j}(t), \\
\alpha_{i}^{3}(s)=\alpha_{i}(s), & \beta_{j}^{3}(t)=\beta_{j}(t),
\end{aligned}
$$

and

$$
Y_{i j}^{1}=Y_{i j}, Y_{i j}^{2}=Y_{i j}^{d}, Y_{i j}^{3}=Y_{i j}^{q},
$$

Propositions 1 and 3 provide for $m=1,2,3$

$$
\begin{aligned}
E\left(Y_{i j}^{m}\right) & =\prod_{s=-\infty}^{\infty} \prod_{t=-\infty}^{\infty}\left(1+b \alpha_{i}^{m}(s) \beta_{j}^{m}(t)\right)^{-a}, \\
E\left(Y_{i j}^{m} Y_{k l}^{m}\right) & =\prod_{s=-\infty}^{\infty} \prod_{t=-\infty}^{\infty}\left(1+b\left[\alpha_{i}^{m}(s) \beta_{j}^{m}(t)+\alpha_{k}^{m}(s) \beta_{l}^{m}(t)\right]\right)^{-a} .
\end{aligned}
$$


The inequality $\ln (1+x) \leq x$ for all $x \geq 0$ yields

$$
\begin{aligned}
& \ln \left(\frac{E\left(Y_{i j}^{m} Y_{k l}^{m}\right)}{E\left(Y_{i j}^{m}\right) E\left(Y_{k l}^{m}\right)}\right) \\
& =\quad \ln \left(\prod_{s=-\infty}^{\infty} \prod_{t=-\infty}^{\infty} \frac{\left(1+b\left[\alpha_{i}^{m}(s) \beta_{j}^{m}(t)+\alpha_{k}^{m}(s) \beta_{l}^{m}(t)\right]\right)^{-a}}{\left(1+b \alpha_{i}^{m}(s) \beta_{j}^{m}(t)\right)^{-a}\left(1+b \alpha_{k}^{m}(s) \beta_{l}^{m}(t)\right)^{-a}}\right) \\
& =\quad a \sum_{s=-\infty}^{\infty} \sum_{t=-\infty}^{\infty} \ln \left(\frac{\left(1+b \alpha_{i}^{m}(s) \beta_{j}^{m}(t)\right)\left(1+b \alpha_{k}^{m}(s) \beta_{l}^{m}(t)\right)}{1+b\left[\alpha_{i}^{m}(s) \beta_{j}^{m}(t)+\alpha_{k}^{m}(s) \beta_{l}^{m}(t)\right]}\right) \\
& \quad a \sum_{s=-\infty}^{\infty} \sum_{t=-\infty}^{\infty} \ln \left(1+\frac{b^{2} \alpha_{i}^{m}(s) \beta_{j}^{m}(t) \alpha_{k}^{m}(s) \beta_{l}^{m}(t)}{1+b\left[\alpha_{i}^{m}(s) \beta_{j}^{m}(t)+\alpha_{k}^{m}(s) \beta_{l}^{m}(t)\right]}\right) \\
& \quad a \sum_{s=-\infty}^{\infty} \sum_{t=-\infty}^{\infty} \ln \left(1+b^{2} \alpha_{i}^{m}(s) \beta_{j}^{m}(t) \alpha_{k}^{m}(s) \beta_{l}^{m}(t)\right) \\
& \leq \quad a \sum_{s=-\infty}^{\infty} \sum_{t=-\infty}^{\infty} b^{2} \alpha_{i}^{m}(s) \beta_{j}^{m}(t) \alpha_{k}^{m}(s) \beta_{l}^{m}(t) .
\end{aligned}
$$

Let

$$
\begin{aligned}
\mathcal{I}^{1} & =\{i ; i=1, \ldots, I\}, \mathcal{J}^{1}=\{j ; j=1, \ldots, J\} \\
\mathcal{I}^{2}= & \{i ; i=1, \ldots, I\}, \mathcal{J}^{2}=\{j ; j=2 n-1 \text { with } n=1, \ldots, J / 2\}, \\
\mathcal{I}^{3}= & \{i ; i=2 n-1 \text { with } n=1, \ldots, I / 2\}, \\
& \quad \mathcal{J}^{3}=\{j ; j=2 n-1 \text { with } n=1, \ldots, J / 2\} .
\end{aligned}
$$

Then for $m=1,2,3$ using Lemma 1 , and noting $\alpha_{i}^{m}(s) \leq \alpha_{i}(s)$ for $i=1, \ldots, I$ and $\beta_{j}^{m}(t) \leq \beta_{j}(t)$ for $j=1, \ldots, J$ we see that

$$
\begin{aligned}
& \sum_{i \in \mathcal{I}^{m}} \sum_{j \in \mathcal{J}^{m}} \sum_{k \in \mathcal{I}^{m}} \sum_{l \in \mathcal{J}^{m}} \ln \left(\frac{E\left(Y_{i j}^{m} Y_{k l}^{m}\right)}{E\left(Y_{i j}^{m}\right) E\left(Y_{k l}^{m}\right)}\right) \\
& \leq a \sum_{i \in \mathcal{I}^{m}} \sum_{j \in \mathcal{J}^{m}} \sum_{k \in \mathcal{I}^{m}} \sum_{l \in \mathcal{J}^{m}} \sum_{s=-\infty}^{\infty} \sum_{t=-\infty}^{\infty} b^{2} \alpha_{i}^{m}(s) \beta_{j}^{m}(t) \alpha_{k}^{m}(s) \beta_{l}^{m}(t) \\
& \leq a \sum_{i=1}^{I} \sum_{j=1}^{J} \sum_{k=1}^{I} \sum_{l=1}^{J} \sum_{s=-\infty}^{\infty} \sum_{t=-\infty}^{\infty} b^{2} \alpha_{i}(s) \beta_{j}(t) \alpha_{k}(s) \beta_{l}(t) \\
& =a b^{2} \sum_{i=1}^{I} \sum_{k=1}^{I} \sum_{s=-\infty}^{\infty} \alpha_{i}(s) \alpha_{k}(s) \sum_{j=1}^{J} \sum_{l=1}^{J} \sum_{t=-\infty}^{\infty} \beta_{j}(t) \beta_{l}(t) \\
& \leq \begin{cases}a b^{2} 12^{2} I J, & \text { for } \rho=0 \\
a b^{2}\left(\frac{1}{\left(1-\rho^{2}\right)(1-\rho)^{4}}\left(4 \cdot 42+\frac{4}{\rho} \cdot 20\right)\right)^{2} I J, & \text { for } \rho \in(0,1)\end{cases} \\
& =c I J
\end{aligned}
$$

where the constant $c$ depends only on $a, b, \rho$. $\square$ 
Proposition 5 For $(a, b, \rho) \in(0, \infty) \times(0, \infty) \times[0,1)$

$$
\begin{array}{ll}
\text { a) } & \operatorname{var}\left(M_{0}\right) \leq c I J, \\
\text { b) } \quad & \operatorname{var}\left(M_{0}^{d}\right) \leq c I J, \\
\text { c) } \quad & \operatorname{var}\left(M_{0}^{q}\right) \leq c I J,
\end{array}
$$

where $c$ is a constant only depending on $a, b$, and $\rho$.

\section{Proof of Proposition 5.}

Using the same notation as in the proof of Proposition 4, we have

$$
M_{0}=\sum_{i \in \mathcal{I}^{1}} \sum_{j \in \mathcal{J}^{1}} Y_{i j}^{1}, M_{0}^{d}=\sum_{i \in \mathcal{I}^{2}} \sum_{j \in \mathcal{J}^{2}} Y_{i j}^{2}, M_{0}^{q}=\sum_{i \in \mathcal{I}^{3}} \sum_{j \in \mathcal{J}^{3}} Y_{i j}^{3} .
$$

Hence for $m=1,2,3$ we have only to prove

$$
\operatorname{var}\left(\sum_{i \in \mathcal{I}^{m}} \sum_{j \in \mathcal{J}^{m}} Y_{i j}^{m}\right) \leq c I J .
$$

First note that

$$
E\left(Y_{i j}^{m}\right)=E\left(Y_{k l}^{m}\right) \quad \text { and } \quad \operatorname{cov}\left(Y_{i j}^{m}, Y_{k l}^{m}\right) \leq \operatorname{var}\left(Y_{i j}^{m}\right)=\operatorname{var}\left(Y_{k l}^{m}\right)
$$

for all $i, k=1, \ldots, I, j, l=1, \ldots, J$, and $m=1,2,3$. This implies

$$
\begin{aligned}
& \frac{\operatorname{var}\left(Y_{11}^{m}\right)}{E\left(Y_{11}^{m}\right)^{2}}=\frac{\operatorname{var}\left(Y_{i j}^{m}\right)}{E\left(Y_{i j}^{m}\right)^{2}} \geq \frac{\operatorname{cov}\left(Y_{i j}^{m}, Y_{k l}^{m}\right)}{E\left(Y_{i j}^{m}\right)^{2}} \\
& =\frac{E\left(Y_{i j}^{m} Y_{k l}^{m}\right)-E\left(Y_{i j}^{m}\right) E\left(Y_{k l}^{m}\right)}{E\left(Y_{i j}^{m}\right) E\left(Y_{k l}^{m}\right)}=\frac{E\left(Y_{i j}^{m} Y_{k l}^{m}\right)}{E\left(Y_{i j}^{m}\right) E\left(Y_{k l}^{m}\right)}-1,
\end{aligned}
$$

so that

$$
\frac{E\left(Y_{i j}^{m} Y_{k l}^{m}\right)}{E\left(Y_{i j}^{m}\right) E\left(Y_{k l}^{m}\right)} \leq \frac{\operatorname{var}\left(Y_{11}^{m}\right)}{E\left(Y_{11}^{m}\right)^{2}}+1=: c_{1}
$$

for all $i, k=1, \ldots, I, j, l=1, \ldots, J$, and $m=1,2,3$. The mean value theorem provides

$$
\begin{aligned}
& \frac{E\left(Y_{i j}^{m} Y_{k l}^{m}\right)}{E\left(Y_{i j}^{m}\right) E\left(Y_{k l}^{m}\right)}-1=\exp \left(\ln \left(\frac{E\left(Y_{i j}^{m} Y_{k l}^{m}\right)}{E\left(Y_{i j}^{m}\right) E\left(Y_{k l}^{m}\right)}\right)\right)-\exp (0) \\
& =\exp \left(\theta_{i j k l}\right)\left(\ln \left(\frac{E\left(Y_{i j}^{m} Y_{k l}^{m}\right)}{E\left(Y_{i j}^{m}\right) E\left(Y_{k l}^{m}\right)}\right)-0\right)
\end{aligned}
$$

with

$$
\theta_{i j k l} \in\left[0, \ln \left(\frac{E\left(Y_{i j}^{m} Y_{k l}^{m}\right)}{E\left(Y_{i j}^{m}\right) E\left(Y_{k l}^{m}\right)}\right)\right] \text { if } \ln \left(\frac{E\left(Y_{i j}^{m} Y_{k l}^{m}\right)}{E\left(Y_{i j}^{m}\right) E\left(Y_{k l}^{m}\right)}\right) \geq 0
$$

and

$$
\theta_{i j k l} \in\left[\ln \left(\frac{E\left(Y_{i j}^{m} Y_{k l}^{m}\right)}{E\left(Y_{i j}^{m}\right) E\left(Y_{k l}^{m}\right)}\right), 0\right] \text { if } \ln \left(\frac{E\left(Y_{i j}^{m} Y_{k l}^{m}\right)}{E\left(Y_{i j}^{m}\right) E\left(Y_{k l}^{m}\right)}\right)<0 .
$$


In particular, $\theta_{i j k l}$ satisfies because of (4)

$$
\theta_{i j k l} \leq \ln \left(\frac{\operatorname{var}\left(Y_{11}^{m}\right)}{E\left(Y_{11}^{m}\right)^{2}}+1\right)=\ln \left(c_{1}\right)
$$

so that

$$
\frac{E\left(Y_{i j}^{m} Y_{k l}^{m}\right)}{E\left(Y_{i j}^{m}\right) E\left(Y_{k l}^{m}\right)}-1 \leq \exp \left(\ln \left(c_{1}\right)\right) \ln \left(\frac{E\left(Y_{i j}^{m} Y_{k l}^{m}\right)}{E\left(Y_{i j}^{m}\right) E\left(Y_{k l}^{m}\right)}\right)=c_{1} \ln \left(\frac{E\left(Y_{i j}^{m} Y_{k l}^{m}\right)}{E\left(Y_{i j}^{m}\right) E\left(Y_{k l}^{m}\right)}\right) .
$$

Then Proposition 4 provides

$$
\begin{aligned}
& \operatorname{var}\left(\sum_{i \in \mathcal{I}^{m}} \sum_{j \in \mathcal{J}^{m}} Y_{i j}^{m}\right) \\
& =\sum_{i \in \mathcal{I}^{m}} \sum_{j \in \mathcal{J}^{m}} \sum_{k \in \mathcal{I}^{m}} \sum_{l \in \mathcal{J}^{m}} \operatorname{cov}\left(Y_{i j}^{m}, Y_{k l}^{m}\right) \\
& =\sum_{i \in \mathcal{I}^{m}} \sum_{j \in \mathcal{J}^{m}} \sum_{k \in \mathcal{I}^{m}} \sum_{l \in \mathcal{J}^{m}} \frac{E\left(Y_{i j}^{m} Y_{k l}^{m}\right)-E\left(Y_{i j}^{m}\right) E\left(Y_{k l}^{m}\right)}{E\left(Y_{i j}^{m}\right) E\left(Y_{k l}^{m}\right)} E\left(Y_{i j}^{m}\right) E\left(Y_{k l}^{m}\right) \\
& =\sum_{i \in \mathcal{I}^{m}} \sum_{j \in \mathcal{J}^{m}} \sum_{k \in \mathcal{I}^{m}} \sum_{l \in \mathcal{J}^{m}}\left(\frac{E\left(Y_{i j}^{m} Y_{k l}^{m}\right)}{E\left(Y_{i j}^{m}\right) E\left(Y_{k l}^{m}\right)}-1\right) E\left(Y_{11}^{m}\right)^{2} \\
& \leq \sum_{i \in \mathcal{I}^{m}} \sum_{j \in \mathcal{J}^{m}} \sum_{k \in \mathcal{I}^{m}} \sum_{l \in \mathcal{J}^{m}} c_{1} \ln \left(\frac{E\left(Y_{i j}^{m} Y_{k l}^{m}\right)}{E\left(Y_{i j}^{m}\right) E\left(Y_{k l}^{m}\right)}\right) E\left(Y_{11}^{m}\right)^{2} \\
& =c_{1} E\left(Y_{11}^{m}\right)^{2} \sum_{i \in \mathcal{I}^{m}} \sum_{j \in \mathcal{J}^{m}} \sum_{k \in \mathcal{I}^{m}} \sum_{l \in \mathcal{J}^{m}} \ln \left(\frac{E\left(Y_{i j}^{m} Y_{k l}^{m}\right)}{E\left(Y_{i j}^{m}\right) E\left(Y_{k l}^{m}\right)}\right) \\
& \leq c_{1} E\left(Y_{11}^{m}\right)^{2} c_{2} I J \\
& =c I J
\end{aligned}
$$

where as $c_{1}, E\left(Y_{11}^{m}\right)^{2}$, and $c_{2}$ only depend on $a, b$, and $\rho$ so does $c$.

\section{Proof of Theorem 1.}

Theorem 1 now follows from Proposition 5 a), b) and c) and Chebyshev's inequality. 